\title{
Biorthogonalité et Théorie des Opérateurs
}

\section{Philippe Tchamitchian}

On dispose maintenant de bases hilbertiennes explicites de $L^{2}\left(\mathbb{R}^{n}\right)$ infiniment plus performantes que la base de Haar. Les fonctions du système de Haar, qui s'écrivent, en dimension 1 ,

$$
h_{j, k}(x)=2^{j / 2} h\left(2^{j} x-k\right),
$$

où

$$
h(x)=\chi_{[0,1 / 2[}(x)-\chi_{[1 / 2,1[}(x) \quad \text { et } j, k \in \mathbb{Z},
$$

si elles sont bien localisées en $x$ sont en revanche discontinues, c'est-à-dire très mal localisées en fréquence, empêchant par là une analyse stable des fonctions. Pierre-Gilles Lemarié et Yves Meyer ont montré qu'on pouvait remplacer la fonction $h$ par une fonction $\psi \in S_{0}(\mathbb{R})$ (l'espace des fonctions de la classe $S$ de Schwartz dont tous les moments sont nuls) et obtenir encore une base hilbertienne de $L^{2}(\mathbb{R})$, et ils ont indiqué comment généraliser à $\mathbb{R}^{n}$ la construction ([9]). Leurs bases sont alors bien localisées à la fois en variable d'espace et en variable de fréquence, et permettent d'analyser avec précision n'importe quel signal: ce sont en fait des bases hilbertiennes de tous les Sobolev, et des bases inconditionnelles de la plupart des espaces fonctionnels classiques.

Il peut dès lors paraître surprenant de s'intéresser aux bases inconditionnelles de $L^{2}\left(\mathbb{R}^{n}\right)$. Cela oblige à étudier des couples de bases inconditionnelles biorthogonales, c'est-à-dire deux systèmes notés pour le moment $\left(\sigma_{\alpha}\right)$ et $\left(\tau_{\alpha}\right)$, 
tels que

$$
\begin{array}{ll}
\forall f \in L^{2}\left(\mathbb{R}^{n}\right) & \int_{\mathbb{R}^{n}}|f|^{2} \sim \sum_{\alpha}\left|\left\langle f, \sigma_{\alpha}\right\rangle\right|^{2} \sim \sum_{\alpha}\left|\left\langle f, \tau_{\alpha}\right\rangle\right|^{2} \\
\forall f \in L^{2}\left(\mathbb{R}^{n}\right) & f=\sum_{\alpha}\left\langle f, \sigma_{\alpha}\right\rangle \tau_{\alpha}=\sum_{\alpha}\left\langle f, \tau_{\alpha}\right\rangle \sigma_{\alpha}
\end{array}
$$

$$
\left\langle\sigma_{\alpha}, \tau_{\beta}\right\rangle=\delta_{\alpha, \beta} .
$$

Mais justement, le fait essentiel que nous présentons dans cet article, et qui ne peut se produire avec une base hilbertienne, est que les deux systèmes $\left(\sigma_{\alpha}\right)$ et $\left(\tau_{\alpha}\right)$ peuvent être très différents de nature. Par exemple, il peut arriver que les $\left(\tau_{\alpha}\right)$ soient des fonctions bien régulières et à supports compacts, tandis que les $\left(\sigma_{\alpha}\right)$ présentent des singularités en certains points. C'est ce qui permet de prouver qu'il ne peut exister de calcul symbolique sur les opérateurs de Calderón-Zygmund (paragraphe VIII).

En nous plaçant au point de vue de l'analyse fonctionnelle, appelons $l_{\sigma_{\alpha}}$ la fonctionnelle qui, à chaque $f \in L^{2}$, associe sa composante suivant le vecteur de base $\sigma_{\alpha}$. Alors, les fonctions $\sigma_{\alpha}$ peuvent être singulières et se comporter plus ou moins comme si elles étaient régulières: les propriétés de régularité sont en fait portées par les fonctionnelles $l_{\sigma_{\alpha}}$. Nous appliquons cela en construisant de nouvelles bases inconditionnelles de $H^{1}\left(\mathbb{R}^{n}\right)$, constituées de fonctions en escalier (paragraphe VII).

Mais pour commencer, nous décrivons un algorithme construisant des couples de bases inconditionnelles biorthogonales de $L^{2}\left(\mathbb{R}^{n}\right)$. Les idées de départ, comme dans [9], sont celles de la transformation en ondelettes (voir [6] et [14]) que nous rappelons maintenant.

Cetains des résultats publiés ici ont déjà paru, sans démonstration, dans la note aux C.R.A.S., citée en référence [15].

\section{Généralités}

Pour le moment, nous restons en dimension 1, le cas de la dimension $n$ faisant l'objet du paragraphe 5 .

Si $g=a x+b, a>0$ et $b \in \mathbb{R}$, est un élément du groupe affine $G$, son action sur une fonction $\sigma \in L^{2}$ donne la fonction $U(g) \sigma$ définie par

$$
U(g) \sigma(x)=a^{1 / 2} \sigma(a x+b) .
$$

Suivant Grossmann, Morlet et Paul ([6]), nous appelons ondelette admissible toute fonction $\sigma$ telle que

$$
C_{\sigma}^{2}=\int_{G}|\langle U(g) \sigma, \sigma\rangle|^{2} d \mu(g)<+\infty,
$$


où $d \mu(g)=d a d b / a$ est la mesure de Haar invariante à droite du groupe $G$. Cette condition est équivalente à la suivante:

$$
\forall \xi \neq 0 \quad \int_{0}^{+\infty}|\hat{\sigma}(a \xi)|^{2} \frac{d a}{a}<+\infty .
$$

Choisissons une autre ondelette admissible $\tau$, et posons

$$
C_{\sigma, \tau}=\frac{1}{\langle\sigma, \tau\rangle} \int_{G}\langle\sigma, U(g) \sigma\rangle \overline{\langle\tau, U(g) \tau\rangle} d \mu(g) .
$$

Dès que $0<\left|C_{\sigma, \tau}\right|<+\infty$, on a la décomposition suivante de l'identité:

$$
\forall f \in L^{2}, \quad f=\frac{1}{C_{\sigma, \tau}} \int_{G}\langle f, U(g) \sigma\rangle U(g) \tau d \mu(g) .
$$

C'est la transformation continue en ondelettes sur $L^{2}$.

Pour obtenir des bases de $L^{2}$, nous commençons par discrétiser la formule précédente, en imposant $a=2^{j}, j \in \mathbb{Z}$, et $b \in \mathbb{Z}$. Si $I$ est l'intervalle dyadique $\left[k 2^{-j},(k+1) 2^{-j}\right]$, où $j, k \in \mathbb{Z}$, nous posons $\sigma_{I}(x)=2^{j / 2} \sigma\left(2^{j} x-k\right)$, et de même pour $\tau$. La notation rappelle que, en un sens à préciser, les fonctions $\sigma_{I}$ et $\tau_{I}$ sont localisées autour de l'intervalle $I$ en variable d'espace, et autour de la bande $1 /(2|I|) \leqslant|\xi| \leqslant 2 /|I|$ en variable de fréquence. Les relations (1) à (3) se réécrivent alors

$$
\begin{gathered}
\int_{\mathbb{R}}|f|^{2} \sim \sum_{I}\left|\left\langle f, \sigma_{I}\right\rangle\right|^{2} \sim \sum_{I}\left|\left\langle f, \tau_{I}\right\rangle\right|^{2}, \\
f=\sum_{I}\left\langle f, \sigma_{I}\right\rangle \tau_{I}=\sum_{I}\left\langle f, \tau_{I}\right\rangle \sigma_{I}, \\
\left\langle\sigma_{I}, \tau_{J}\right\rangle=\delta_{I, J}
\end{gathered}
$$

oú il est entendu une fois pour toutes que $I$ et $J$ parcourent l'ensemble des intervalles dyadiques.

La relation (2) est la discrétisation de (5). Il faut remarquer que, parce que les $g_{j, k}=2^{j} x-k$ ne forment pas un sous-groupe du groupe affine, il n'est pas du tout nécessaire que le système biorthogonal aux $\sigma_{I}$ soit constitué de l'orbite d'une seule fonction $\tau$ sous l'action des $g_{j, k}$. C'est une hypothèse que nous imposons pour nous permettre de calculer.

Une deuxième remarque, beaucoup plus importante, est à faire. C'est la relation (1) qui fixe le cadre fonctionnel dans lequel on opère, ici $L^{2}$ et $l^{2}$. La relation (2) peut avoir un sens dans d'autres espaces fonctionnels que $L^{2}$, d'autant plus que la convergence des séries écrites peut être forte ou faible. Nous reviendrons sur ce point quand nous sortirons du cadre $L^{2}$. Quant à (3), elle est pratiquement vide de tout contenu fonctionnel, et a un sens dès que 
le crochet $\langle\sigma, \tau\rangle$ existe. Tout ceci a des répercussions cruciales sur l'invariance des relations (1) à (3).

Si $L$ est un opérateur borné inversible sur $L^{2}$, en écrivant $f=L^{-1} L f=L L^{-1} f$, on voit que les relations (1) à (3) restent vraies pour les familles $\left(L^{*}\left(\sigma_{I}\right)\right)$ et $\left(L^{-1}\left(\tau_{I}\right)\right)$, ou $\left(L\left(\sigma_{I}\right)\right)$ et $\left(L^{*-1}\left(\tau_{I}\right)\right)$.

En revanche, cela ne peut être le cas si $L$ est non borné, à cause de (1). Supposons cependant que $L$ ait un domaine dense, soit inversible sur son image, et surtout qu'il soit invariant par translation et jouisse d'une certaine propriété d'homogénéité:

$$
\forall t>0, \quad L(\varphi(t x))=t^{\alpha} L \varphi(t x)
$$

pour un certain $\alpha$ (par exemple $\left.L=D, L=\sqrt{-\Delta}, L=D^{\alpha}\right)$. Dans ce cas, les relations (2) et (3) sont formellement vraies pour les familles $\left(L^{*} \sigma\right)_{I}$ et $\left(L^{-1} \tau\right)_{I}$ dès que $L^{*} \sigma$ et $L^{-1} \tau$ existent. Pour qu'elles aient un sens dans $L^{2}$, il reste à s'assurer que

$$
\int_{\mathbb{R}}|f|^{2} \sim \sum_{I}\left|\left\langle f,\left(L^{*} \sigma\right)_{I}\right\rangle\right|^{2} \sim \sum_{I}\left|\left\langle f,\left(L^{-1} \tau\right)_{I}\right\rangle\right|^{2} .
$$

En particulier, si les fonctions $\sigma$ et $\tau$ engendrent deux bases biorthogonales de $L^{2}$, pour certaines valeurs de $\alpha$, on aura de nouvelles bases à partir des fonctions $D^{\alpha} \sigma$ et $D^{-\alpha} \tau$ : c'est pourquoi deux bases biorthogonales peuvent être de régularités très différentes.

Enfin, soulignons que les relations (1), (2) et (3) ne sont pas indépendantes. Pour le voir, il est nécessaire de faire un rappel sur la notion de «frame».

On appelle «frame» une famille de fonctions $\left(\psi_{\alpha}\right)$ telle que, pour toute $f \in L^{2}$, on ait

$$
\int|f|^{2} \sim \sum_{\alpha}\left|\left\langle f, \psi_{\alpha}\right\rangle\right|^{2}
$$

Dans [4], Ingrid Daubechies montre qu'un frame est nécessairement une partie génératrice, au sens qu'il existe un autre frame $\left(\tilde{\psi}_{\alpha}\right)$ tel que, si $f \in L^{2}$,

$$
f=\sum_{\alpha}\left\langle f, \tilde{\psi}_{\alpha}\right\rangle \psi_{\alpha}=\sum_{\alpha}\left\langle f, \psi_{\alpha}\right\rangle \tilde{\psi}_{\alpha} .
$$

Cela vient de ce que l'opérateur $A$ qui, à $f \in L^{2}$, associe les coefficients $\left\langle f, \psi_{\alpha}\right\rangle$, est inversible sur son image. On en déduit que $A^{*} A$ est inversible sur $L^{2}$, et on pose alors $\tilde{\psi}_{\alpha}=\left(A^{*} A\right)^{-1} \psi_{\alpha}$.

Ce résultat implique immédiatement que les relations (1) à (3) sont équivalentes aux deux relations

$$
\int_{\mathbb{R}}|f|^{2} \sim \sum_{I}\left|\left\langle f, \sigma_{I}\right\rangle\right|^{2} \text { et }\left\langle\sigma_{I}, \tau_{J}\right\rangle=\delta_{I, J}
$$


Cette réduction des équations n'est cependant pas très utile dans la pratique, car pour prouver l'inégalité

$$
\int_{\mathbb{R}}|f|^{2} \leqslant C \sum_{I}\left|\left\langle f, \sigma_{I}\right\rangle\right|^{2},
$$

on est souvent obligé de passer par (1), et de prouver ensuite que

$$
\sum_{I}\left|\left\langle f, \tau_{I}\right\rangle\right|^{2} \leqslant C \int_{\mathbb{R}}|f|^{2} .
$$

Au paragraphe suivant, nous expliquons pourquoi il est plus facile de démontrer une inégalité dans ce sens.

\section{Un Algorithme de Construction de Bases Inconditionnelles de $L^{2}(\mathbb{R})$}

Nous rentrons maintenant dans le vif du sujet, en prouvant le:

Théorème 1. Soit $g$ une fonction continue $4 \pi$ - périodique telle que $g(\xi)>0$ si $\xi \in] 0,4 \pi\left[\right.$, et $g(\xi)=0\left(\xi^{2 p}\right)$ au voisinage de 0 . On a normalisé $g$ en imposant $g(2 \pi)=1$.

Soient

$$
p(\xi)=\frac{g(\xi+2 \pi)}{g(\xi)+g(\xi+2 \pi)}
$$

et

$$
G(\xi)=\frac{1}{g(\xi)+g(\xi+2 \pi)} \prod_{j=1}^{+\infty} g\left(2^{-j} \xi\right) .
$$

On suppose qu'il existe $q \in[1, p]$ tel que $\left(1+\xi^{2}\right)^{q} G(\xi) \in L^{\infty}$.

Définissons les fonctions $\sigma$ et $\tau$ par

$$
\hat{\sigma}(\xi)=e^{-i \xi / 2} \frac{g(\xi)}{|\xi|^{q}} \quad \text { et } \quad \hat{\tau}(\xi)=e^{-i \xi / 2}|\xi|^{q} G(\xi) .
$$

Alors, les familles $\left(\sigma_{I}\right)$ et $\left(\tau_{I}\right)$ forment un couple de bases inconditionnelles biorthogonales de $L^{2}(\mathbb{R})$.

On peut remplacer dans cet énoncé $|\xi|^{q} \operatorname{par} \operatorname{sgn}(\xi)|\xi|^{q}$ : cela revient à utiliser l'invariance du problème par la transformation de Hilbert. Nous étudierons au paragraphe 6 la question plus délicate de savoir pour quelles valeurs de $\alpha$ 
les familles $\left(D^{\alpha} \sigma\right)_{I}$ et $\left(D^{-\alpha} \tau\right)_{I}$ sont encore des couples de bases de $L^{2}(\mathbb{R})$. Nous donnerons également, après la démonstration du théorème, des exemples qui montrent que les hypothèses peuvent être satisfaites pour des valeurs de $p$ et $q$ arbitrairement grandes (paragraphe 3). Pour le moment, nous faisons simplement remarquer que ce théorème redonne le système de Haar, à partir de $g(\xi)=\sin ^{2} \xi / 4$, et $p=q=2$.

Démontrons le théorème, c'est-à-dire les relations (1), (2) et (3) pour $\sigma$ et $\tau$ définies par (6).

Supposons pour commencer avoir montré (2) pour toute $f \in S_{0}(\mathbb{R})$ (espace des fonctions de la classe de Schwartz dont tous les moments sont nuls) et (3). Nous obtenons (2) pour toute $f \in L^{2}(\mathbb{R})$ et (1) grâce au lemme suivant, d'un usage constant dans la théorie:

Lemme 1. Soit $\sigma$ une ondelette et $\nu$ un réel tels que

$$
\sum_{I}|I|^{\nu}\left|\left\langle\sigma, \sigma_{I}\right\rangle\right|<+\infty
$$

on a alors les deux inégalités

$$
\begin{aligned}
& \forall\left(\lambda_{I}\right) \in l^{2}, \quad \int_{\mathbb{R}}\left|\sum_{I} \lambda_{I} \sigma_{I}\right|^{2} \leqslant C \sum_{I}\left|\lambda_{I}\right|^{2}, \\
& \forall f \in L^{2}(\mathbb{R}), \quad \sum_{I}\left|\left\langle f, \sigma_{I}\right\rangle\right|^{2} \leqslant C \int_{\mathbb{R}}|f|^{2}
\end{aligned}
$$

En effert par changement de variable, la condition (7) implique l'existence d'une constante $C>0$ telle que, pour tout intervalle $J$,

$$
\sum_{I}\left(\frac{|I|}{|J|}\right)^{\nu}\left|\left\langle\sigma_{J}, \sigma_{I}\right\rangle\right| \leqslant C .
$$

On en déduit (8) en écrivant:

$$
\begin{aligned}
\sum_{I} \sum_{J} \lambda_{I} \bar{\lambda}_{J}\left\langle\sigma_{J}, \sigma_{I}\right\rangle \leqslant & \left(\sum_{I}\left|\lambda_{I}\right|^{2} \sum_{J}\left(\frac{|J|}{|I|}\right)^{\nu}\left|\left\langle\sigma_{I}, \sigma_{J}\right\rangle\right|\right)^{1 / 2} \\
& \left(\sum_{J}\left|\lambda_{J}\right|^{2} \sum_{I}\left(\frac{|I|}{|J|}\right)^{\nu}\left|\left\langle\sigma_{I}, \sigma_{J}\right\rangle\right|\right)^{1 / 2} \\
& \leqslant C \sum_{I}\left|\lambda_{I}\right|^{2} .
\end{aligned}
$$

Quant à l'inégalité (9), c'est la version adjointe de la précédente.

Un critère très utile dans la pratique pour vérifier (7) est le suivant 
Lemme 2. Soit $\sigma$ une fonction telle que, pour un certain $\delta>0$,

$$
\begin{gathered}
|\sigma(x)| \leqslant C\left(1+x^{2}\right)^{-(1+\delta / 2)}=C \omega_{\delta}(x), \\
\left|\sigma(x)-\sigma\left(x^{\prime}\right)\right| \leqslant C\left|x-x^{\prime}\right|^{\delta}\left[\omega_{\delta}(x)+\omega_{\delta}\left(x^{\prime}\right)\right], \\
\int_{\mathbb{R}} \sigma(x) d x=0 .
\end{gathered}
$$

Alors, pour tout $\nu$ tel que $\left|\nu-\frac{1}{2}\right|<\delta$, o vérifie (7).

La preuve est laissée au lecteur. Le lemme est d'ailleurs classique en théorie des intégrales singulières: voir Lemarié [8] ou Meyer [12], par exemple.

Les lemmes 2 et 1 s'appliquent immédiatement aux ondelettes $\sigma$ et $\tau$ définies par (6). On en déduit (1) et (2) pour toute $f \in L^{2}$, si on l'a prouvé pour $f \in S_{0}(\mathbb{R})$.

Soit donc $f \in S_{0}(\mathbb{R})$, et posons

$$
f_{j}=\sum_{|I|=2-j}\left\langle f, \sigma_{I}\right\rangle \tau_{I},
$$

c'est-à-dire

$$
f_{j}(t)=\sum_{k} 2^{j}\left(\int f(u) \sigma\left(2^{j} u-k\right) d u\right) \tau\left(2^{j} t-k\right) .
$$

Traduisons cela en variable de Fourier:

$$
\hat{f}_{j}(\xi)=\sum_{k} 2^{-j} \frac{1}{2 \pi} \int \hat{f}(\eta) e^{i k 2^{-j} \eta} \overline{\hat{\sigma}\left(2^{-j} \eta\right)} d \eta e^{-i k 2^{-j_{\xi}}} \hat{\tau}\left(2^{-j} \xi\right) .
$$

La formule de Poisson s'applique, et donne

$$
\hat{f}_{j}(\xi)=\sum_{k \in \mathbb{Z}} \hat{f}\left(\xi+2 \pi k 2^{j}\right) \overline{\hat{\sigma}\left(2^{-j} \xi+2 \pi k\right)} \hat{\tau}\left(2^{-j} \xi\right) .
$$

En sommant sur $j$ et en regroupant les termes, on obtient

$$
\begin{aligned}
\sum_{j \in \mathbb{Z}} \hat{f}_{j}(\xi)= & \hat{f}(\xi) \sum_{j \in \mathbb{Z}} \overline{\hat{\sigma}\left(2^{-j} \xi\right)} \hat{\tau}\left(2^{-j} \xi\right) \\
& +\sum_{j \in \mathbb{Z}} \sum_{k_{0} \in 2 \mathbb{Z}+1} \hat{f}\left(\xi+2 \pi k_{0} 2^{j}\right) \sum_{n \geq 0} \overline{\hat{\sigma}\left(2^{n}\left[2^{-j} \xi+2 \pi k_{0}\right]\right)} \hat{\tau}\left(2^{n-j} \xi\right)
\end{aligned}
$$

(il faut remarquer que tout nombre dyadique $k 2^{j}$ se décompose de manière unique en $k_{0} 2^{j_{0}}$, où $k_{0}$ est impair et $j_{0}=j+n$ pour un certain $n \geqslant 0$ ).

Cette égalité est valable pour toute $f \in S_{0}$. On est donc ramené à prouver

$$
\forall \xi \neq 0, \quad \sum_{j \in \mathbb{Z}} \overline{\hat{\sigma}\left(2^{-j} \xi\right)} \hat{\tau}\left(2^{-j} \xi\right)=1,
$$


et

(15) $\forall \xi$ et $\forall k_{0}$ impair, si $\xi \neq-2 \pi k_{0}$

$$
\sum_{n \geq 0} \overline{\hat{\sigma}\left(2^{n}\left[\xi+2 \pi k_{0}\right]\right)} \hat{\tau}\left(2^{n} \xi\right)=0 .
$$

On remplace maintenant $\hat{\sigma}$ et $\hat{\tau}$ par leurs définitions. Grâce au choix des phases et à la $4 \pi$-périodicité de la fonction $g$, le système infini précédent se réduit au couple d'équations suivantes:

$$
\begin{aligned}
& \forall \xi \neq 0, \quad \sum_{j \in \mathbb{Z}} g\left(2^{-j} \xi\right) G\left(2^{-j} \xi\right)=1 \\
& \forall \xi \neq 0, \quad \sum_{n \geq 1} g\left(2^{n} \xi\right) G\left(2^{n} \xi\right)=g(\xi+2 \pi) G(\xi) .
\end{aligned}
$$

En fait, il suffit de vérifier (17), car, en l'appliquant à $2^{-N} \xi$, on obtient

$$
\sum_{j \geq-N+1} g\left(2^{j} \xi\right) G\left(2^{j} \xi\right)=g\left(2^{-N} \xi+2 \pi\right) G\left(2^{-N} \xi\right)
$$

La limite de cette égalité quand $N$ tend vers $+\infty$ donne exactement (16). Quant à (17), elle se déduit de l'équation fonctionnelle satisfaite par $g$ et $G$ :

$$
G(\xi) g(\xi+2 \pi)=G(2 \xi)[g(2 \xi)+g(2 \xi+2 \pi)] .
$$

En itérant cette relation, et en tenant compte de ce que $\lim _{\xi \rightarrow \pm \infty} G(\xi)=0$ on prouve (17), et par conséquent (2) pour toute $f \in S_{0}$.

Il reste enfin à prouver (3). Là encore, on utilise la formule de Poisson pour montrer que (3) est équivalent au système d'équations suivant, où $j \geqslant 1$ :

$$
\sum_{k \in \mathbb{Z}} \overline{\hat{\sigma}(\xi+2 \pi k)} \hat{\tau}\left(2^{j}[\xi+2 \pi k]\right)=0 \text { pour tout } \xi,
$$

$$
\sum_{k \in \mathbb{Z}} \overline{\hat{\sigma}\left(2^{j}[\xi+2 \pi k]\right)} \hat{\tau}(\xi+2 \pi k)=0 \text { pour tout } \xi,
$$

$$
\sum_{k \in \mathbb{Z}} \overline{\hat{\sigma}(\xi+2 \pi k)} \hat{\tau}(\xi+2 \pi k)=1 \text { pour tout } \xi .
$$

En fonction de $g$ et $G$, ces équations deviennent:

$$
\begin{aligned}
\left(A_{j}\right) & \sum_{k \in \mathbb{Z}}(-1)^{k} g(\xi+2 \pi k) G\left(2^{j}[\xi+2 \pi k]\right) & =0, \\
\left(B_{j}\right) & \sum_{k \in \mathbb{Z}}(-1)^{k} g\left(2^{j}[\xi+2 \pi k]\right) G(\xi+2 \pi k) & =0, \\
\text { (C) } & \sum_{k \in \mathbb{Z}} g(\xi+2 \pi k) G(\xi+2 \pi k) & =1 .
\end{aligned}
$$

La clé est le lemme suivant: 
Lemme 3.

$$
H(\xi)=\sum_{k \in \mathbb{Z}} G(\xi+4 \pi k)=\frac{1}{g(\xi)+g(\xi+2 \pi)} \quad \text { pour tout } \xi
$$

Supposons-le acquis. On prouve $(C)$ de la manière suivante:

$$
\begin{aligned}
\sum_{k \in \mathbb{Z}} g(\xi+2 \pi k) G(\xi+2 \pi k) & =\sum_{k \text { pair }}+\sum_{k \text { impair }} \\
& =g(\xi) H(\xi)+g(\xi+2 \pi) H(\xi+2 \pi) \\
& =1 .
\end{aligned}
$$

Les équations $\left(B_{j}\right)$ deviennent

$$
\begin{aligned}
\sum_{k \in \mathbb{Z}}(-1)^{k} g\left(2^{j}[\xi+2 \pi k]\right) G(\xi+2 \pi k) & =g\left(2^{j} \xi\right) \sum_{k \in \mathbb{Z}}(-1)^{k} G(\xi+2 \pi k) \\
& =g\left(2^{j} \xi\right)[H(\xi)-H(\xi+2 \pi)] \\
& =0 .
\end{aligned}
$$

La preuve des $\left(A_{j}\right)$ est à peine moins simple. On commence par $\left(A_{1}\right)$ :

$$
\begin{aligned}
\sum_{k \in \mathbb{Z}}(-1)^{k} g(\xi+2 \pi k) & G(2 \xi+4 \pi k) \\
& =g(\xi) \sum_{k \text { pair }} G(2 \xi+4 \pi k)-g(\xi+2 \pi k) \sum_{k \text { impair }} G(2 \xi+4 \pi k),
\end{aligned}
$$

et on utilise la relation (18), qu'on écrit par commodité sous la forme

$$
G(2 \xi)=H(2 \xi) g(\xi+2 \pi) G(\xi)
$$

Cela donne

$$
\begin{aligned}
\sum_{k \in \mathbb{Z}}(-1)^{k} g(\xi & +2 \pi k) G(2 \xi+4 \pi k) \\
& =H(2 \xi) g(\xi) g(\xi+2 \pi) H(\xi)-H(2 \xi) g(\xi+2 \pi) g(\xi) H(\xi+2 \pi)=0 .
\end{aligned}
$$

Ensuite, si $j \geqslant 2$, on écrit

$$
G\left(2^{j}[\xi+2 \pi k]\right)=H\left(2^{j} \xi\right) g\left(2^{j} \xi+2 \pi\right) G\left(2^{j-1}[\xi+2 \pi k]\right),
$$

ce qui montre que $\left(A_{j-1}\right) \Rightarrow\left(A_{j}\right)$.

Pour achever la démonstration du théorème 1 , prouvons le lemme 3 . Il est équivalent à l'identité suivante:

$$
S(\xi)=\sum_{k \in \mathbb{Z}} R(\xi+4 \pi k)=1,
$$


où l'on a posé

$$
R(\xi)=\prod_{j=1}^{+\infty} p\left(2^{-j} \xi\right) \quad\left(\text { et } \quad p(\xi)=\frac{g(\xi+2 \pi)}{g(\xi)+g(\xi+2 \pi)}\right) .
$$

On a $R(2 \xi)=p(\xi) R(\xi)$, et $p(\xi)+p(\xi+2 \pi)=1, p(\xi)>0$ si $\xi \in]-2 \pi, 2 \pi[$. On en déduit

$$
\begin{aligned}
S(2 \xi) & =\sum_{k \in \mathbb{Z}} p(\xi+2 \pi k) R(\xi+2 \pi k) \\
& =p(\xi) \sum_{k \text { pair }} R(\xi+2 \pi k)+p(\xi+2 \pi) \sum_{k \text { impair }} R(\xi+2 \pi k),
\end{aligned}
$$

ce qui donne l'équation fonctionnelle

$$
S(2 \xi)=p(\xi) S(\xi)+p(\xi+2 \pi) S(\xi+2 \pi) .
$$

$S$ étant $4 \pi$-périodique, nous nous restreignons à [ $-2 \pi, 2 \pi]$. Si $S$ atteint son maximum en $\xi_{0} \in[-2 \pi, 2 \pi]$, alors $S$ doit l'atteindre aussi en $\xi_{0} / 2$, et donc, par itération, en $\xi_{0} / 2^{n}$ pour tout $n$, et en 0 à la limite: on a prouvé $S \leqslant 1$.

Le même raisonnement vaut pour les minima de $S$, ce qui conclut la démonstration du lemme 3 et du théorème 1 .

\section{Deux Familles D'exemples}

PREMIER EXEMPLE: BASES D'ONDELETTES À SUPPORTS COMPACTS. Si, dans la construction du théorème 1 , on veut que les fonctions $\sigma$ et $\tau$ soient à supports compacts, alors il faut imposer à la fonction $g$ une condition supplémentaire. Il faut que $g$ soit un polynôme trigonométrique, et que $g(\xi)+g(\xi+2 \pi)=1$. A ce moment-là, le produit infini $G$ s'écrit $G(\xi)=\Pi g\left(2^{-j} \xi+2 \pi\right)$ : c'est une fonction entière de type exponentiel, égal à celui de $g$.

Une classe générale d'exemples est décrite ci-dessous.

Lemme 4. Soit l'ensemble

$A=\{P \in \mathbb{R}[X] ; \quad P(0)=0, \quad P(u)>0$ si $0<u \leqslant 1, \quad P(u)+P(1-u)=1\}$.

Si $P \in A$, soient $p$ la valuation de $P$ et

$$
B=\sup _{u \in[0,1]} \frac{P(u)}{u^{p}} .
$$

On pose

$$
g(\xi)=P\left(\sin ^{2} \xi / 4\right) \quad \text { et } \quad G(\xi)=\prod_{j=1}^{+\infty} g\left(2^{-j} \xi+2 \pi\right) .
$$


Alors, si

$$
q=p-\frac{\log B}{\log 4}, \quad\left(1+\xi^{2}\right)^{q} G(\xi) \in L^{\infty} .
$$

Soit $\xi \geqslant 2$, et $j_{0}$ tel que $2^{-j_{0}-1} \xi \leqslant 1 \leqslant 2^{-j_{0}} \xi$. Alors,

$$
\begin{aligned}
G(\xi) & \leqslant \prod_{j=1}^{j_{0}} g\left(2^{-j} \xi+2 \pi\right) \\
& =\prod_{j=1}^{j_{0}} P\left(\cos ^{2} 2^{-j} \xi / 4\right) \\
& \leqslant B^{j_{0}} \prod_{j=1}^{j_{0}}\left(\cos ^{2} 2^{-j} \xi / 4\right)^{p} .
\end{aligned}
$$

Comme

$$
\prod_{j=1}^{\infty} \cos 2^{-j} u=\frac{\sin u}{u}
$$

on a

$$
G(\xi) \leqslant B^{j_{0}}\left(\frac{\sin \xi / 4}{\xi / 4}\right)^{2 p}\left(\inf _{|u| \leq 2} \frac{\sin u}{u}\right)^{-2 p} .
$$

Or, $j_{0} \leqslant \log \xi / \log 2$, donc

$$
G(\xi) \leqslant C \frac{\left(\sin ^{2} \xi / 4\right)^{p}}{\xi^{2 p-\log B / \log 2}} .
$$

Construisons maintenant une suite de polynômes $P_{n} \in A$ telle que les constantes associées

$$
q_{n}=p_{n}-\frac{\log B_{n}}{\log 4}
$$

tendent vers l'infini.

L'ensemble $A$ jouit de la propriété suivante: si $P, Q \in A$, alors $P \circ Q \in A$.

D'autre part, $P_{1}(u)=3 u^{2}-2 u^{3} \in A$.

Posons alors $P_{n}=P_{1} \circ \ldots \circ P_{1}$. La valuation de $P_{n}$ est $p_{n}=2^{n}$, et un calcul donne $B_{n}=3^{2^{n}-1}$. On a donc

$$
q_{n}=2^{n}\left(1-\frac{\log 3}{\log 4}\right)+\frac{\log 3}{\log 4} .
$$


Si, à $n$ fixé, on prend $q=\left[q_{n}\right]$, les fonctions $\sigma$ et $\tau$ définies par (6), avec $g_{n}, G_{n}$ et $q$, sont à supports compacts et $(q-2)$ fois dérivables. La fonction $\sigma$ est en fait une fonction spline, et $\sigma^{(q-2)}$ est lipschitzienne. La fonction $\tau$ est plus compliquée: on peut seulement affirmer que

$$
\left|\tau^{(q-2)}(x+h)+\tau^{(q-2)}(x-h)-2 \tau^{(q-2)}(x)\right| \leqslant C|h| .
$$

Au paragraphe 7, nous revenons plus en détail sur les fonctions obtenues avec le polynôme $P_{1}$.

DeUXIÈME EXEMPLE: LES BASES HILBERTIENNES D'ONDELETTES DE LEMARIÉ ET Battle. Chacun de leur côté et indépendamment de nous, P. G. Lemarié, puis $\mathrm{G}$. Battle ont construit des bases orthonormales d'ondelettes, à localisation exponentielle et de régularité finie, mais arbitrairement grande.

C'est leur construction qui nous a suggéré de rechercher une version du théorème publié dans [15] un peu plus forte, qui puisse expliquer les similitudes troublantes qui apparaissent. C'est ainsi que nous avons abouti au théorème 1.

Pour obtenir les bases de Lemarié et Battle, il suffit de résoudre l'équation

$$
G(\xi)=\frac{g(\xi)}{\xi^{2 m}}
$$

où $m \geqslant 1$ est fixé, et $G$ est défini comme d'habitude à partir de $g$. En utilisant le lemme 3 , le lecteur pourra se convaincre que les seules solutions sont données par

$$
g(\xi)=\frac{A_{m}(\xi+2 \pi)}{A_{m}(\xi)+A_{m}(\xi+2 \pi)},
$$

où

$$
A_{m}(\xi)=\sum_{k} \frac{1}{(\xi+4 \pi k)^{2 m}}
$$

Là encore, les ondelettes obtenues (avec $q=m)$ sont $(m-2)$ fois dérivables, et $\sigma^{(m-2)}=\tau^{(m-2)}$ est une fonction lipschitzienne, $\sigma$ est en fait une fonction-spline sur tout $\mathbb{R}$ : sur chaque intervalle $[k / 2,(k+1) / 2], k \in \mathbb{Z}, \sigma$ est un polynôme de degré $m-1$.

Nous ne savons pas s'il existe des bases orthonormales d'ondelettes à supports compacts et de régularité arbitrairement grande. En tout cas, l'algorithme du théorème 1 n'en permet pas la construction.

Nous allons maintenant passer au cas de la dimension $n$. Comme dans [9], ce passage dépend du calcul des sommes partielles en dimension 1 . 


\section{Cacul des Sommes Partielles en Dimension 1}

Soient, si $f \in L^{2}(\mathbb{R})$,

$$
S_{0} f=\sum_{|I| \geq 2}\left\langle f, \sigma_{I}\right\rangle \tau_{I} \quad \text { et } \quad S_{N} f=\sum_{|I| \geq 2-N+1}\left\langle f, \sigma_{I}\right\rangle \tau_{I} .
$$

Si $\delta_{N}$ est l'opérateur de dilatation par $2^{N}: \delta_{N} f(x)=f\left(2^{N} x\right)$ alors $S_{N}=\delta_{N} S_{0} \delta_{-N}$. Pour calculer les $S_{N}$ il suffit donc de calculer $S_{0}$.

Repartons de (13): en sommant sur les indices $j \leqslant-1$, on obtient

$$
\left(S_{0} f\right)^{\wedge}(\xi)=\sum_{j \leq-1} \sum_{k} \hat{f}\left(\xi+2 \pi k 2^{j}\right) \overline{\hat{\sigma}\left(2^{-j} \xi+2 \pi k\right)} \hat{\tau}\left(2^{-j} \xi\right) .
$$

On écrit alors les entiers $k$ sous la forme $2^{n} k_{0}$, où $n \geqslant 0$ et $k_{0}$ impair

$$
\begin{aligned}
\left(S_{0} f\right)^{\wedge}(\xi)= & \sum_{j \leq 1} \hat{f}(\xi) \overline{\hat{\sigma}\left(2^{-j} \xi\right)} \hat{\tau}\left(2^{-j} \xi\right) \\
& +\sum_{j \leq 1} \sum_{n \geq 0} \sum_{\substack{k_{0} \\
\text { impair }}} \hat{f}\left(\xi+2 \pi 2^{j+n} k_{0}\right) \overline{\hat{\sigma}\left(2^{-j} \xi+2 \pi 2^{n} k_{0}\right)} \hat{\tau}\left(2^{-j} \xi\right) \\
= & \sum_{j \leq 1} \hat{f}(\xi) \overline{\hat{\sigma}\left(2^{-j} \xi\right)} \hat{\tau}\left(2^{-j} \xi\right) \\
& +\sum_{j \in \mathbb{Z}} \sum_{\substack{k_{0} \\
\text { impair }}} \hat{f}\left(\xi+2 \pi 2^{j} k_{0}\right)\left(\sum_{\substack{n \geq 0 \\
n \geq j+1}} \overline{\hat{\sigma}\left(2^{n}\left[2^{-j} \xi+2 \pi k_{0}\right]\right)} \hat{\tau}\left(2^{n-j} \xi\right)\right.
\end{aligned} .
$$

D'après (15), le terme entre parenthèses est nul si $j+1 \leqslant 0$. Il ne reste donc que les indices $j \in \mathbb{N}$ dans la dernière somme. Ecrivant $2^{j} k_{0}=k$, on en déduit:

$$
\left(S_{0} f\right)^{\wedge}(\xi)=\sum_{k \in \mathbb{Z}} \hat{f}(\xi+2 \pi k)\left[\sum_{j \geq 1} \overline{\hat{\sigma}\left(2^{j}[\xi+2 \pi k]\right)} \hat{\tau}\left(2^{j} \xi\right)\right] .
$$

Grâce à (17), on calcule la somme entre crochets:

$$
\begin{aligned}
\sum_{j \geq 1} \overline{\hat{\sigma}\left(2^{j}[\xi+2 \pi k]\right)} \hat{\tau}\left(2^{j} \xi\right) & =\frac{|\xi|^{q}}{|\xi+2 k \pi|^{q}} \sum_{j \geq 1} g\left(2^{j}[\xi+2 \pi k]\right) G\left(2^{j} \xi\right) \\
& =\frac{|\xi|^{q}}{|\xi+2 \pi k|^{q}} \sum_{j \geq 1} g\left(2^{j} \xi\right) G\left(2^{j} \xi\right) \\
& =\frac{|\xi|^{q}}{|\xi+2 \pi k|^{q}} g(\xi+2 \pi) G(\xi) .
\end{aligned}
$$

On vérifie que ce calcul est vrai même si $\xi+2 \pi k=0$ ou $\xi=0$.

Soient les fonctions $\varphi$ et $\gamma$ définies par

$$
\hat{\varphi}(\xi)=\left|\frac{\sin \xi / 2}{\xi / 2}\right|^{q} \quad \text { et } \quad \hat{\gamma}(\xi)=\left|\frac{\xi / 2}{\sin \xi / 2}\right|^{q} g(\xi+2 \pi) G(\xi) .
$$


Alors, pour tous $\xi$ et $k$,

$$
\hat{\varphi}(\xi+2 \pi k) \hat{\gamma}(\xi)=\frac{|\xi|}{|\xi+2 \pi k|^{q}} g(\xi+2 \pi) G(\xi) .
$$

Une nouvelle application de (13) montre que

$$
S_{0} f=\sum_{|I|=1}\left\langle f, \varphi_{I}\right\rangle \gamma_{I} .
$$

Une dernière propriété, importante pour la suite, est que $\left\langle\varphi_{I}, \gamma_{J}\right\rangle=\delta_{I, J} \mathrm{si}$ $|I|=|J|$. En effet, on a

$$
\begin{aligned}
\sum_{k} \overline{\hat{\varphi}(\xi+2 \pi k)} \hat{\gamma}(\xi+2 \pi k) & =\sum_{k} g(\xi+2 \pi k+2 \pi) G(\xi+2 \pi k) \\
& =g(\xi+2 \pi) H(\xi)+g(\xi) H(\xi+2 \pi) \\
& =1 .
\end{aligned}
$$

Les définitions de $\varphi$ et $\gamma$ peuvent être modifiées, en particulier pour mieux s'adapter aux ondelettes des exemples du paragraphe précédent (et sans rien changer aux propriétés qu'on vient de démontrer).

Si l'on veut que $\varphi$ et $\gamma$ soient à supports compacts, $q$ étant alors un entier, on pose

$$
\hat{\varphi}(\xi)=e^{-i q \xi / 2}\left(\frac{\sin \xi / 2}{\xi / 2}\right)^{q}
$$

et

$$
\hat{\gamma}(\xi)=e^{-i q \xi / 2}\left(\frac{\xi / 2}{\sin \xi / 2}\right)^{q} g(\xi+2 \pi) G(\xi)
$$

Si l'on veut, dans le cas où $G(\xi)=g(\xi) / \xi^{2 m}$, avoir $\varphi=\gamma$, il faut prendre

$$
\hat{\varphi}(\xi)=\hat{\gamma}(\xi)=\frac{\sqrt{g(\xi) g(\xi+2 \pi)}}{\xi^{m}} .
$$

Cela montre d'ailleurs que les fonctions $\varphi$ et $\gamma$ définissant les opérateurs de sommes partielles suivant (21) ne sont pas uniques.

Enfin, remarquons que la fonction $\varphi$, définie dans (20), est indépendante de $g$ et de $G$. Dans le cas où $q$ est entier, c'est un $B$-spline ( $B$ pour basic), égal à la convolution de la fonction caractéristique de $[-1 / 2,1 / 2] q$ fois avec ellemême. 


\section{Bases d'Ondelettes en Dimension $n$}

Nous imitons dans ce paragraphe la construction donnée dans [9].

La collection des intervalles dyadiques est maintenant remplacée par celle des cubes dyadiques. $Q$ est un cube dyadique si on a $Q=I_{1} \times I_{2} \times \cdots \times I_{n}$, où les $I_{j}$ sont des intervalles dyadiques de même longueur: $Q$ est donc défini par un indice $j \in \mathbb{Z}$ et un multi-indice $k=\left(k_{1}, k_{2}, \ldots, k_{n}\right)$. Si $\psi \in L^{2}\left(\mathbb{R}^{n}\right)$, on pose $\psi_{Q}(x)=2^{n j / 2} \psi\left(2^{j} x-k\right)$.

Soit (en dimension 1) $D_{j}$ la projection sur les $\tau_{I}$ pour $|I|=2^{-j}$ et $E_{j}$ la projection sur les $\gamma_{I}$ pour les mêmes intervalles. Cela signifie que

$$
D_{j} f=\sum_{|I|=2-j}\left\langle f, \sigma_{i}\right\rangle \tau_{I} \text { et } E_{j} f=\sum_{|I|=2-j}\left\langle f, \varphi_{I}\right\rangle \gamma_{I} .
$$

Le calcul des sommes partielles se résume par l'égalité

$$
D_{j}=E_{j+1}-E_{j} .
$$

Passons en dimension 2. On a alors

$$
D_{j} \otimes D_{j}+D_{j} \otimes E_{j}+E_{j} \otimes D_{j}=E_{j+1} \otimes E_{j+1}-E_{j} \otimes E_{j} .
$$

Notons $\sigma^{0}=\sigma, \sigma^{1}=\varphi, \tau^{0}=\tau$ et $\tau^{1}=\gamma$. Soient les six ondelettes de $\mathbb{R}^{2}$ définies par $\sigma^{\left(\epsilon_{1}, \epsilon_{2}\right)}=\sigma^{\epsilon_{1}} \otimes \sigma^{\epsilon_{2}}$ et $\tau^{\left(\epsilon_{1}, \epsilon_{2}\right)}=\tau^{\epsilon_{1}} \otimes \tau^{\epsilon_{2}}$, où $\left(\epsilon_{1}, \epsilon_{2}\right)=(0,0),(0,1)$ et $(1,0)$. La relation (23) implique

$$
\forall f \in L^{2}\left(\mathbb{R}^{2}\right) \quad f=\sum_{Q} \sum_{\epsilon}\left\langle f, \sigma_{Q}^{\epsilon}\right\rangle \tau_{Q}^{\epsilon},
$$

$Q$ parcourant l'ensemble des cubes dyadiques, et $\epsilon=\left(\epsilon_{1}, \epsilon_{2}\right)$.

On vérifie ensuite que les families $\left(\sigma_{Q}^{\epsilon}\right)$ et $\left(\tau_{Q}^{\epsilon}\right)$ sont biorthogonales.

Pour cela, il faut remarquer que $S_{0} \gamma=\gamma$, ce qui s'écrit

$$
\sum_{|I| \geq 2}\left\langle\gamma, \sigma_{I}\right\rangle \tau_{I}=\sum_{I}\left\langle\gamma, \sigma_{I}\right\rangle \tau_{I}
$$

Par conséquent, $\left\langle\gamma, \sigma_{I}\right\rangle=0$ si $|I| \leqslant 1$. On prouve de la même façon les relations suivantes:

$$
\left\langle\gamma_{I}, \sigma_{J}\right\rangle=0 \text { si }|J| \leqslant|I| \text { et }\left\langle\varphi_{I}, \tau_{J}\right\rangle=0 \text { si }|J| \leqslant|I| .
$$

Jointes aux égalités déjà connues:

$$
\left\langle\sigma_{I}, \tau_{J}\right\rangle=\delta_{I, J} \text { et }\left\langle\varphi_{I}, \gamma_{J}\right\rangle=\delta_{I, J} \text { si }|I|=|J|,
$$

on en déduit $\left\langle\sigma_{Q}^{\epsilon}, \tau_{Q^{\prime}}^{\epsilon^{\prime}}\right\rangle=\delta_{Q, Q^{\prime}} \delta_{\epsilon, \epsilon^{\prime}}$. 
Enfin, les lemmes 1 et 2 se généralisant sans problème, on prouve comme en dimension 1 que, si $f \in L^{2}$,

$$
\int_{\mathbb{R}^{2}}|f|^{2} \sim \sum_{Q} \sum_{\epsilon}\left|\left\langle f, \sigma_{Q}^{\epsilon}\right\rangle\right|^{2} \sim \sum_{Q} \sum_{\epsilon}\left|\left\langle f, \tau_{Q}^{\epsilon}\right\rangle\right|^{2} .
$$

Les choses se passent bien sûr de la même façon en dimension $n \geqslant 3$. On a donc le théorème suivant:

Théorème 2. Avec les notations du théorème 1 et du paragraphe 4, soit $\sigma^{0}=\sigma, \sigma^{1}=\varphi, \tau^{0}=\tau, \tau^{1}=\gamma$. Soit E l'ensemble des indices $\epsilon=\left(\epsilon_{1}, \ldots, \epsilon_{n}\right)$, où $\epsilon_{i}=0$ ou 1 et l'un au moins des $\epsilon_{i}$ est nul. Définissons $2^{n+1}-2$ ondelettes de $\mathbb{R}^{n}$ par les formules $\sigma^{\epsilon}=\sigma^{\epsilon_{1}} \otimes \cdots \otimes \sigma^{\epsilon_{n}}, \tau^{\epsilon}=\tau^{\epsilon_{1}} \otimes \cdots \otimes \tau^{\epsilon_{n}}$.

Alors, les $\left(\sigma_{Q}^{\epsilon}\right)$ et les $\left(\tau_{Q}^{\epsilon}\right)$, où $Q$ parcourt l'ensemble des cubes dyadiques, forment un couple de bases inconditionnelles biorthogonales de $L^{2}\left(\mathbb{R}^{n}\right)$.

Nous disposons maintenant d'une classe de bases de $L^{2}\left(\mathbb{R}^{n}\right)$, de régularités finies et arbitrairement grandes. Etudions ce qui se passe quand on fait varier la régularité, c'est-à-dire quand le couple $(\sigma, \tau)$ est remplacé par le couple $\left(D^{\alpha} \sigma, D^{-\alpha} \tau\right)$.

\section{Nouvelles Bases de $L^{2}$ Obtenues en Intégrant par Parties}

Nous nous cantonnons en dimension 1, laissant au lecteur le passage à la dimension $n$.

Ensuite, nous fixons l'exposant $q$ qui intervient dans le théorème 1 et la définition des ondelettes $\sigma$ et $\tau$ en imposant les conditions $\left(1+\xi^{2}\right)^{q-\epsilon} G(\xi) \in L^{\infty}$ et $\left(1+\xi^{2}\right)^{q+\epsilon} G(\xi) \notin L^{\infty}$ pour tout $\epsilon>0$.

Notre problème est la détermination des valeurs de $\alpha$ pour lesquelles les familles $\left(D^{\alpha} \sigma\right)_{I}$ et $\left(D^{-\alpha} \tau\right)_{I}$ sont encore des bases de $L^{2}(\mathbb{R})$. Ce qui revient à demander: pour quelles valeurs de $\alpha$ a-t-on

$$
\forall f \in L^{2}(\mathbb{R}) \quad \int_{\mathbb{R}}|f|^{2} \sim \sum_{I}\left|\left\langle f,\left(D^{\alpha} \sigma\right)_{I}\right\rangle\right|^{2} \sim \sum_{I}\left|\left\langle f,\left(D^{-\alpha} \tau\right)_{I}\right\rangle\right|^{2} ?
$$

If faut naturellement que $D^{\alpha} \sigma$ et $D^{-\alpha} \tau$ soient dans $L^{2}(\mathbb{R})$, ce qui impose $|\alpha|<q-1 / 2$. Il se trouve que cette condition est suffisante si l'on rajoute certaine hypothèse sur la fonction $g$.

Théorème 3. Avec les notations du théorème 1, on suppose que

$$
\sum_{n \in \mathbb{Z}} n^{2}|\hat{g}(n / 2)|<+\infty .
$$


Alors les familles $\left(D^{\alpha} \sigma\right)_{I}$ et $\left(D^{-\alpha} \tau\right)_{I}$ forment un couple de bases biorthogonales de $L^{2}(\mathbb{R})$ dès que $D^{\alpha} \sigma \in L^{2}(\mathbb{R})$ et $D^{-\alpha} \tau \in L^{2}(\mathbb{R})$.

Les relations (2) et (3) étant formellement stables par intégration par parties, le théorème se ramène à montrer que, si $|\alpha|<q-1 / 2$, alors

$$
\sum_{I}\left|\left\langle f,\left(D^{\alpha} \sigma\right)_{I}\right\rangle\right|^{2} \leqslant C \int_{\mathbb{R}}|f|^{2}
$$

et de même

$$
\sum_{I}\left|\left\langle f,\left(D^{-\alpha} \tau\right)_{I}\right\rangle\right|^{2} \leqslant C \int_{\mathbb{R}}|f|^{2} .
$$

Dans le cas où $|\alpha|<q-1$, les fonctions $D^{\alpha} \sigma$ et $D^{-\alpha} \tau$ vérifient toutes les deux les hypothèses du lemme 2, et il n'y a rien de plus à démontrer.

Plaçons-nous d'abord dans le cas $q-1<\alpha<q-1 / 2$, c'est-à-dire celui où

$$
\left(D^{\alpha} \sigma\right)^{\wedge}(\xi)=e^{-i \xi / 2} \frac{g(\xi)}{|\xi|^{1 / 2+\epsilon}}
$$

pour un certain $\epsilon \in] 0,1 / 2\left[\right.$. Nous allons prouver que $D^{\alpha} \sigma$ vérifie l'inégalité (7) avec $\nu=0$ (alors que $D^{\alpha} \sigma$ ne satisfait pour aucun $\delta>0$ les hypothèses du lemme 2):

$$
\sum_{|I| \leq 1}\left|\left\langle D^{\alpha} \sigma,\left(D^{\alpha} \sigma\right)_{I}\right\rangle\right|<+\infty .
$$

Cela suffira, car $D^{-\alpha} \tau$ est bien régulière et décroissante à l'infini: on lui applique le lemme 2 .

On a

$$
g(\xi)=\sum_{n} a_{n} e^{i n \xi / 2},
$$

et

$$
\sum_{n} n^{2}\left|a_{n}\right|<+\infty .
$$

La somme écrite dans (26) devient

$$
\begin{aligned}
\sum_{j \geq 0} \sum_{k} 2^{j / 2}\left|\int_{\mathbb{R}} e^{i k \xi}\left(D^{\alpha} \sigma\right)^{\wedge}\left(2^{j} \xi\right) \overline{\left(D^{\alpha} \sigma\right)^{\wedge}(\xi)} d \xi\right|= \\
\quad=\sum_{j \geq 0} \sum_{k} 2^{-j \epsilon}\left|\int_{\mathbb{R}} e^{i\left(k+(1 / 2)-2^{j-1}\right) \xi} \frac{g(\xi) g\left(2^{j} \xi\right)}{|\xi|^{1+2}} d \xi\right| \\
\quad \leqslant \sum_{n}\left|a_{n}\right| \sum_{j \geq 0} 2^{-j \epsilon} \sum_{k}\left|\int_{\mathbb{R}} e^{i\left(k+(1 / 2)+(n-1) 2^{j-1}\right) \xi} \frac{g(\xi)}{|\xi|^{+2 \epsilon}} d \xi\right| .
\end{aligned}
$$


Tout se ramène donc à montrer

$$
\sum_{k}\left|\int_{\mathbb{R}} e^{i k \xi} \frac{g(\xi)}{|\xi|^{1+2 \epsilon}} d \xi\right| \leqslant C \quad(\text { pour } j=0)
$$

et

$$
\left.\sum_{k}\left|\int_{\mathbb{R}} e^{i(k+(1 / 2)) \xi} \frac{g(\xi)}{|\xi|^{1 / 2 \epsilon}} d \xi\right| \leqslant C \quad \text { (pour } j \geqslant 1\right) .
$$

Or,

$$
\int_{\mathbb{R}} e^{i k \xi} \frac{g(\xi)}{|\xi|^{1+2 \epsilon}} d \xi=C \sum_{n} a_{n}|k+n / 2|^{2} .
$$

On utilise alors la qualité du zéro de $g$ en 0: on a au moins $g(\xi)=0\left(\xi^{2}\right)$, donc $\sum_{n} a_{n}=\sum_{n} n a_{n}=0$. Avec l'hypothèse (25) cela implique

$$
\sum_{n} a_{n}|k+n / 2|^{2 \epsilon}=0\left(|k|^{2 \epsilon-2}\right)
$$

à l'infini, et $2 \epsilon-2<-1$ permet de conclure.

Le cas $\alpha=q-1$ peut se traiter suivant les mêmes idées, ou bien aussi par interpolation. Comme nous développerons ce point dans le paragraphe suivant, nous laissons ce cas au lecteur.

Il reste enfin le cas $-q+1 / 2<\alpha \leqslant-q+1$, qui se traite en utilisant la dualité entre les espaces de Beppo-Levi. Rappelons que l'espace $B^{\alpha}$ est défini par la condition $\int_{\mathbb{R}}|\hat{f}(\xi)|^{2}|\xi|^{2}<+\infty$, et que $B^{\alpha}$ et $B^{-\alpha}$ sont duaux l'un de l'autre.

Soient les deux opérateurs

$$
S f=\sum_{I}\left\langle f, \sigma_{I}\right\rangle \sigma_{I} \quad \text { et } \quad T f=\sum_{I}\left\langle f, \tau_{I}\right\rangle \tau_{I} .
$$

Nous venons de prouver la continuité de $S$ sur $B^{\alpha}$, pour tout $\alpha$ dans l'intervalle $]-q+1 / 2,-q+1]$. En effet, cette continuité est équivalente à celle de l'opérateur $D^{\alpha} S D^{-\alpha}$ sur $L^{2}$, qui s'écrit

$$
D^{\alpha} S D^{-\alpha} f=\sum_{I}\left\langle f,\left(D^{-\alpha} \sigma\right)_{I}\right\rangle\left(D^{\alpha} \sigma\right)_{I} .
$$

La fonction $D^{\alpha} \sigma$ vérifiant les conditions du lemme 2 , on a

$$
\int_{\mathbb{R}}\left|D^{\alpha} S D^{-\alpha} f\right|^{2} \leqslant C \sum_{I}\left|\left\langle f,\left(D^{-\alpha} \sigma\right)_{I}\right\rangle\right|^{2} \leqslant C \int_{\mathbb{R}}|f|^{2}
$$

d'après ce qui précède. 
De même, nous avons prouvé que $T$ est continu sur $B^{-\alpha}$. Comme $S$ et $T$ sont auto-adjoints, ils sont continus sur $B^{-\alpha}$ et $B^{\alpha}$ respectivement. Ainsi, $D^{-\alpha} S D^{\alpha}$ est continu sur $L^{2}$, et comme les $\left(D^{-\alpha} \sigma\right)_{I}$ forment une base de $L^{2}$, cela veut dire que

$$
\sum_{I}\left|\left\langle f,\left(D^{\alpha} \sigma\right)_{I}\right\rangle\right|^{2} \leqslant C \int_{\mathbb{R}}|f|^{2}
$$

De même,

$$
\sum_{I}\left|\left\langle f,\left(D^{-\alpha} \tau\right)_{I}\right\rangle\right|^{2} \leqslant C \int_{\mathbb{R}}|f|^{2},
$$

et le théorème est démontré.

Les valeurs limites de $\alpha$ montrent bien les différences qui peuvent apparaître entre deux bases biorthogonales associées.

Prenons par exemple $\alpha=q-1 / 2-\epsilon, 0<\epsilon<1 / 2$. Alors,

$$
\left(D^{\alpha} \sigma\right)(x)=0\left(\left|x-\frac{n+1}{2}\right|^{-1 / 2+\epsilon}\right)
$$

au voisinage de $(n+1) / 2$, si $g(n / 2) \neq 0 . D^{\alpha} \sigma$ n'est donc dans $L_{\text {loc }}^{p}(\mathbb{R})$ que si $p<1 /(1 / 2-\epsilon)$. En revanche, $D^{-\alpha} \tau$ est une fonction suffisamment douce et régulière pour vérifier $\left|D^{-\alpha} \tau(x)\right|+\left|D^{-\alpha+1} \tau(x)\right| \leqslant C(1+|x|)^{-3 / 2-\epsilon}$.

Dans l'autre cas limite $\alpha=-q+1 / 2-\epsilon, 0<\epsilon<1 / 2, D^{-\alpha} \tau \in H^{s}$ seulement pour $s<\epsilon$, tandis que $D^{\alpha} \sigma \in H^{s}$ pour $s<4 q-2 \epsilon$.

Le cas $\alpha=q-1$ est également intéressant, il permet d'obtenir une base inconditionnelle de $H^{1}$ (l'espace de Stein et Weiss) constituée de fonctions en escalier.

\section{Bases Inconditionnelles sur les $L^{p}, H^{1}$ et BMO}

Dans ce paragraphe, nous voulons décrire, dans l'échelle des espaces $H^{1}, L^{p}$ et BMO, ceux pour lesquels les familles de fonctions que nous venons de construire sont des bases. Pour l'espace BMO, qui n'est pas séparable, cela doit être entendu au sens faible de la dualité avec $H^{1}$. Les autres espaces sont munis de leur topologie forte. Là encore, nous restons en dimension 1.

La théorie des opérateurs de Calderón-Zygmund intervient ici de façon cruciale.

Rappelons qu'un noyau de Calderón-Zygmund est une fonction $K(x, y)$ définie sur $\mathbb{R} \times \mathbb{R}$ privé de sa diagonale, et telle que

$$
|K(x, y)| \leqslant \frac{C}{|x-y|},
$$




$$
\begin{aligned}
& \left|K(x, y)-K\left(x^{\prime}, y\right)\right| \leqslant C \frac{\left|x-x^{\prime}\right|^{\delta}}{|x-y|^{1+\delta}} \quad \text { si } \quad\left|x-x^{\prime}\right| \leqslant \frac{1}{2}|x-y|, \\
& \left|K(x, y)-K\left(x, y^{\prime}\right)\right| \leqslant C \frac{\left|y-y^{\prime}\right|^{\delta}}{|x-y|^{1+\delta}} \quad \text { si } \quad\left|y-y^{\prime}\right| \leqslant \frac{1}{2}|x-y|,
\end{aligned}
$$

pour un certain $\delta \in] 0,1]$ et une certaine constante $C$.

Un opérateur de Calderón-Zygmund (CZO en abrégé) est un opérateur continu sur $L^{2}$ et dont le noyau est de Calderón-Zygmund. $\dagger$ Un CZO est automatiquement continu sur les $L^{p}, 1<p<+\infty$, de $H^{1}$ dans $L^{1}$, de BMO dans $L^{\infty}$, et envoie $L^{1}$ dans $L^{1}$ faible. Il est continu sur $H^{1}$ si $T^{*}(1)=0(T$ est le nom de l'opérateur), et continu sur BMO si $T(1)=0$. Les CZO $T$ tels que $T(1)=T^{*}(1)=0$ forment une algèbre, découverte et étudiée par Lemarié ([2], [7] et [8]).

Pour nous, le point important est le suivant:

Lemme 5. Soient deux ondelettes $\psi$ et $\tilde{\psi}$ vérifiant les hypothèses du lemme 2 . Alors l'opérateur A défini par

$$
A f=\sum_{i}\left\langle f, \psi_{I}\right\rangle \tilde{\psi}_{I}
$$

est un CZO appartenant à l'algèbre de Lemarié.

Les estimations sur le noyau de $A$ viennent de (10) et (11), la continuité sur $L^{2}$ de $A$ vient du lemme 2, et l'appartenance à l'algèbre de Lemarié vient de (12).

Revenons à nos familles $\left(D^{\alpha} \sigma\right)_{I}$ et $\left(D^{-\alpha} \sigma\right)_{I}$, et supposons pour commencer que $|\alpha|<q-1$.

Soit $\left(\psi_{I}\right)$ une base d'Yves Meyer: $\psi \in S_{0}(\mathbb{R})$ et $\left(\psi_{I}\right)$ est une base des $L^{p}$, $1<p<+\infty$, de $H^{1}$ et de BMO, entre autres ([9]). Alors, les deux opérateurs suivants

$$
S_{\alpha} f=\sum_{I}\left\langle f, \psi_{I}\right\rangle\left(D^{\alpha} \sigma\right)_{I}
$$

et

$$
T_{\alpha} f=\sum_{I}\left\langle f,\left(D^{-\alpha} \tau\right)_{I}\right\rangle \psi_{I}
$$

sont des CZO inverses l'un de l'autre. Ils sont donc continus sur les $L^{p}$, $1<p<+\infty$, sur $H^{1}$ et sur BMO, et inversibles. Cela implique que les $\left(D^{\alpha} \sigma\right)_{I}$ et les $\left(D^{-\alpha} \tau\right)_{I}$ sont des bases inconditionnelles biorthogonales de ces espaces.

Supposons maintenant que $\alpha=q-1 / 2-\epsilon, 0<\epsilon<1 / 2$. Nous réservons $\alpha=q-1$. Alors, $T_{\alpha}$ reste un CZO, mais pas $S_{\alpha}$. On a vu en effet que $D^{\alpha} \sigma \in$ $L_{\text {loc }}^{p}$ seulement si $p<1 /(1 / 2-\epsilon)$.

Si $\alpha=-q+1 / 2+\epsilon, 0<\epsilon<1 / 2$, c'est $T_{\alpha}$ qui cesse d'être un CZO.

Dans ces deux cas, le résultat est le suivant: 
Théorème 4. Avec les notations précédentes, les $\left(D^{\alpha} \sigma\right)_{I}$ et les $\left(D^{-\alpha} \tau\right)_{I}$ sont des bases inconditionnelles biorthogonales de $L^{p}$ si et seulement si $|1 / p-1 / 2|<\epsilon$, c'est-à-dire si et seulement si $D^{\alpha} \sigma$ et $D^{-\alpha} \tau$ appartiennent à $L^{p} \cap L^{p^{\prime}}, p$ et $p^{\prime}$ étant conjugués.

Démontrons ce théorème quand $\alpha=q-1 / 2-\epsilon$. La nécessité de la condition est évidente. Pour la réciproque, nous prouvons que, si $|1 / p-1 / 2|<\epsilon$, $S_{\alpha}$ est continu sur $L^{p}$. Comme $T_{\alpha}$ est un CZO, cela permet de conclure.

La continuité de $S_{\alpha}$ sur $L^{p}$ se montre par interpolation complexe. Ecrivons $\alpha=(1-\lambda) \alpha_{1}+\lambda \alpha_{2}$, où $\alpha<\alpha_{1}<q-1 / 2$ et $\alpha_{2}<q-1$. Alors, l'ensemble des $p$ pour lesquels il existe $\left.p_{1} \in\right] 1,+\infty[$ et $\lambda \in] 0,2 \in[$ tels que

$$
\frac{1}{p}=\frac{1-\lambda}{2}+\frac{\lambda}{p_{1}}
$$

est exactement l'intervalle $] \frac{1}{1 / 2+\epsilon}, \frac{1}{1 / 2-\epsilon}[$.

Soit

$$
a=\frac{p_{1}-2}{p_{1}\left(\alpha-\alpha_{2}\right)+2\left(\alpha_{1}-\alpha\right)} .
$$

Si $z \in \mathbb{C}$ et $\alpha_{2} \leqslant \operatorname{Re}(z) \leqslant \alpha_{1}$, posons

$$
S_{z} f=e^{z^{2}-\alpha^{2}} \sum_{I}\left\langle f_{Z}, \psi_{I}\right\rangle\left(\sigma_{z}\right)_{I},
$$

où tout à fait classiquement, si $f=|f| e^{i \theta}, f_{z}=|f|^{\partial(z-\alpha)+1} e^{i \theta}$, et où

$$
\hat{\sigma}_{z}(\xi)=|\xi|^{z} \hat{\sigma}(\xi)=e^{-i \xi / 2}|\xi|^{z-q} g(\xi)
$$

On vérifie alors successivement que

1) $\operatorname{Si} \operatorname{Re}(z)=\alpha_{1}, S_{z}$ est uniformément borné sur $L_{2}$.

2) Si $\operatorname{Re}(z)=\alpha_{2}, S_{z}$ est uniformément borné sur $L_{2}$, et $S_{z}$ est un CZO, dont les constantes de Calderón-Zygmund (appelées $C$ et $\delta$ dans (27), (28) et (29)) sont elles aussi uniformément bornées, grâce au terme $e^{z^{2}-\alpha^{2}}$. On en déduit que $S_{z}$ est uniformément borné sur $L^{p_{1}}$.

3) Si $f, g \in S_{0}$, alors $\left\langle S_{z} f, g\right\rangle$ est holomorphe dans un voisinage de la bande $\alpha_{2} \leqslant \operatorname{Re}(z) \leqslant \alpha_{1}$.

Cela implique que $S_{\alpha}=S$ est borné sur $L^{p}$.

On procède de la même façon quand $\alpha=-q+1 / 2+\epsilon$, en échangeant les rôles de $\sigma$ et $\tau$. Le théorème est démontré. 
Le cas $\alpha=q-1$ est spécialement intéressant. Les $\left(D^{\alpha} \sigma\right)_{I}$, qui sont des fonctions en escalier, sont, par ce qui précède, une base inconditionnelle de tous les $L^{p}$. En cela, elles ressemblent au système de Haar.

EXEMPLe. Soit $g(\xi)=P_{1}\left(\sin ^{2} \xi / 4\right)$, où $P_{1}(u)=3 u^{2}-2 u^{3}$, et $s$ définie par $\hat{s}(\xi)=e^{-i \xi / 2}(g(\xi) / \xi)$. La fonction $s$ est la fonction en escalier à support compact dont le graphe est représenté ci-dessous.

Mais il y a pourtant une grande différence entre la famille des $\left(\sigma_{I}\right)$, où $s=D^{q-1} \sigma$, et le système de Haar, qui réside dans la régularité du système biorthogonal associé. Cela a pour conséquence le

Théorème 5. Soit $g$ vérifiant les hypothèses du théorème 3 , et $s$ la fonction en escalier définie par $\hat{s}(\xi)=e^{-i \xi / 2}(g(\xi) / \xi)$. Alors, les $\left(\sigma_{I}\right)$ forment une base inconditionnelle de $H^{1}(\mathbb{R})$.

Par dualité, ce théorème se ramène à l'énoncé suivant: $f \in \mathrm{BMO}(\mathbb{R})$ si et seulement si, pour tout intervalle $I$, on a

$$
\sum_{J \subset I}\left|\left\langle f, s_{J}\right\rangle\right|^{2} \leqslant C|I|
$$

la plus petite des constantes étant équivalente à $\|f\|_{\text {BMO }}^{2}$. Car, si $\left(t_{I}\right)$ est le système biorthogonal associé aux $\left(s_{I}\right)$, cela veut dire que $\left(t_{I}\right)$ est une base inconditionnelle (au sens faible) de BMO.

Supposons pour commencer $f \in \mathrm{BMO}$, et $m_{I} f=0$. Par changement de variable, il suffit de montrer (30) quand $I=[0,1]$. Posons, si $l \geqslant 1, \chi_{l}=\chi_{2^{l} \leq|x| \leq 2^{l+1}}$, $f_{l}=f \chi_{l}$, et $f_{0}=f \chi_{\{|x| \leq 2\}}$. Grâce au théorème 3 , on a 


$$
\sum_{J \subset[0,1]}\left|\left\langle f_{0}, s_{J}\right\rangle\right|^{2} \leqslant \int_{\mathbb{R}}\left|f_{0}\right|^{2} \leqslant C\|f\|_{\text {BMO }}^{2} .
$$

Si $l \geqslant 1$, on écrit

$$
\sum_{J \subset[0,1]}\left|\left\langle f_{l}, s_{J}\right\rangle\right|^{2}=\left\langle P f_{l}, f_{l}\right\rangle \leqslant\left\|\chi_{l} P \chi_{l}\right\|_{2,2}\left\|f_{l}\right\|_{2}^{2},
$$

où $P$ est l'opérateur défini par

$$
P f=\sum_{J \subset[0,1]}\left\langle f, s_{J}\right\rangle s_{J} .
$$

Puisque $f \in \mathrm{BMO},\left\|f_{l}\right\|_{2}^{2} \leqslant C\left(1+l^{2}\right) 2^{l}\|f\|_{\text {BMO }}^{2}$. D'autre part, le lemme de Schur montre que

$$
\left\|\chi_{l} P \chi_{l}\right\|_{2,2} \leqslant \sup _{K \subset[0,1]} \sum_{J \subset[0,1]}\left|\left\langle\chi_{l} s_{J}, s_{K}\right\rangle\right| .
$$

La majoration du membre de droite est un peu longue, mais sans difficulté spéciale. Nous nous contentons donc d'indiquer la marche à suivre. Il suffit d'écrire explicitement la fonction $s$.

$\mathrm{Si}$

$$
g(\xi)=\sum_{n} a_{n} e^{i n \xi / 2},
$$

et si

$$
r_{p}=\sum_{n \leq p} a_{n},
$$

on a

$$
s(x)=\sum_{p} r_{p} \chi_{\{(p-1) / 2 \leq x \leq p / 2\}},
$$

et

$$
\sum_{p} p\left|r_{p}\right|<+\infty .
$$

On calcule $s_{j}$ et $s_{k}$, puis $\left\langle\chi_{l} s_{j}, s_{k}\right\rangle$. On majore brutalement $\sum_{J \subset[0,1]}\left|\left\langle\chi_{l} s_{j}, s_{k}\right\rangle\right|$, en tenant compte des localisations imposées par la présence de $\chi_{l}$ et les conditions $J \subset[0,1], K \subset[0,1]$. Il vient alors $\left\|\chi_{l} P \chi_{l}\right\|_{2,2} \leqslant C 2^{-2 l}$.

Par conséquent,

$$
\sum_{J \subset[0,1]}\left|\left\langle f_{l}, s_{J}\right\rangle\right|^{2} \leqslant C\left(1+l^{2}\right) 2^{-l}\|f\|_{\text {BMO }}^{2},
$$

d'où le résultat désiré

$$
\sum_{J \subset[0,1]}\left|\left\langle f, s_{J}\right\rangle\right|^{2} \leqslant C\|f\|_{\mathrm{BMO}}^{2} .
$$


Supposons maintenant que, pout tout intervalle $I$, on ait

$$
\sum_{J \subset I}\left|\left\langle f, s_{J}\right\rangle\right|^{2} \leqslant C|I|
$$

On montre que $f \in \mathrm{BMO}$ en construisant un CZO, $U$, tel que $f=U(1)$. Pour cela, si $I=\left[k 2^{-j},(k+1) 2^{-j}\right]$, et si $\varphi$ est une fonction bosse $(\varphi \in D(\mathbb{R}), \varphi \geqslant 0$ et $\int \varphi=1$ ), on pose $\varphi_{I}(x)=2^{j} \varphi\left(2^{j} x-k\right)$ (noter la différence de normalisation). L'opérateur $U$ est le paraproduit (voir [13]) donné par le noyau

$$
U(x, y)=\sum_{I}\left\langle f, s_{I}\right\rangle t_{I}(x) \varphi_{I}(y),
$$

où $\left(t_{I}\right)$ est, rappelons-le, la famille biorthogonale associée aux $\left(s_{I}\right)$. Il est connu que la condition (30) implique la continuité de $U$ sur $L^{2}$ ([13]). $U$ est un CZO grâce à la condition plus faible $\left|\left\langle f, s_{I}\right\rangle\right| \leqslant C|I|^{1 / 2}$, et surtout grâce à la régularité et à la décroissance des fonctions $t_{I}$. Donc, $U(1) \in \mathrm{BMO}$. On vérifie facilement que $U(1)=f$ au sens des distributions, ce qui achève de prouver le théorème.

Nous terminons cette étude banachique par l'espace $L^{1}$ : il n'admet pas de bases inconditionnelles, cela est bien connu. Mais on a:

Théorème 6. Soient $\sigma$ et $\tau$ comme au théorème 1 , et soient $\varphi$ et $\gamma$ les fonctions associées aux sommes partielles décrites au paragraphe 1 .

Alors, les deux systèmes $\left(\sigma_{I}\right)_{|I| \leq 1},\left(\varphi_{I}\right)_{|I|=2}$ et $\left(\tau_{I}\right)_{|I| \leq 1},\left(\gamma_{I}\right)_{|I|=2}$ forment un couple de bases conditionnelles biorthogonales de $L^{1}(\mathbb{R})$. De plus, si

$$
f=\sum_{|I| \leq 1} \lambda_{I} \sigma_{I}+\sum_{|I|=2} \lambda_{I} \varphi_{I} \in L^{1} \quad \text { et } \quad \epsilon_{I}= \pm 1
$$

alors

$$
f_{\epsilon}=\sum_{|I| \leq 1} \epsilon_{I} \lambda_{I} \sigma_{I}+\sum_{|I| \leq 1} \epsilon_{I} \lambda_{I} \varphi_{I}
$$

est dans l'espace $L_{\text {faible }}^{1}$, et de même si $\sigma$ et $\varphi$ sont remplacées par $\tau$ et $\gamma$.

Rappelons que $L_{\text {faible }}^{1}$ est l'espace des fonctions telles que, pour tout $\lambda>0$, $|\{x:|f(x)|>\lambda\}| \leqslant C / \lambda$.

Montrons que les $\left(\sigma_{I}\right)_{|I| \leq 1}$ et $\left(\varphi_{I}\right)_{|I|=2}$ sont une base de $L^{1}$. Il faut voir pourquoi, si $f \in L^{1}$,

$$
\lim _{N \rightarrow \infty}\left\|f-\sum_{2-N-1 \leq|I| \leq 1} \lambda_{I} \sigma_{I}-\sum_{|I|=2} \lambda_{I} \varphi_{I}\right\|_{1}=0,
$$

où $\lambda_{I}=\left\langle f, \tau_{I}\right\rangle$ si $|I| \leqslant 1$ et $\lambda_{I}=\left\langle f, \gamma_{I}\right\rangle$ si $|I|=2$. Mais cela s'écrit

$$
\lim _{N \rightarrow+\infty}\left\|f-\sum_{|I|=2-N}\left\langle f, \gamma_{I}\right\rangle \varphi_{I}\right\|_{1}=0 .
$$


Or, on vérifie que $\varphi(2 \pi k)=\delta_{0, k}$ donc $\sum_{k} \varphi(x-k)=1$. Par conséquent

$f(x)-\sum_{|I|=2-N}\left\langle f, \gamma_{I}\right\rangle \varphi_{I}(x)=\sum_{k} 2^{N} \varphi\left(2^{N} x-k\right) \int_{\mathbb{R}}[f(x)-f(t)] \gamma\left(2^{N} t-k\right) d t$

d'où

$$
\begin{aligned}
\left\|f-\sum_{|I|=2-N}\left\langle f, \gamma_{I}\right\rangle \varphi_{I}\right\|_{1} & \leqslant \iint|f(x)-f(t)| \sum_{k} 2^{N}\left|\gamma\left(2^{N} t-k\right)\right| \varphi\left(2^{N} x-k\right) \mid d t d x . \\
& \leqslant C \iint|f(x)-f(t)| \frac{2^{N}}{1+\left(2^{N}|t-x|\right)^{2}} d t d x
\end{aligned}
$$

Il est maintenant classique de conclure par (31).

Il reste à voir pourquoi $f_{\epsilon} \in L_{\text {faible }}^{1}$ : cela vient de la théorie des CZO. En effet, $f_{\epsilon}=Y_{\epsilon}(f)$, où $Y_{\epsilon}$ est de noyau

$$
\sum_{|I| \leq 1} \epsilon_{I} \sigma_{I}(x) \tau_{I}(y)+\sum_{|I|=2} \epsilon_{I} \varphi_{I}(x) \gamma_{I}(y) .
$$

$Y_{\epsilon}$ est continu sur $L^{2}$ d'après les paragraphes 2 et 4 , et son noyau est de Calderón-Zygmund. Donc, $Y_{\epsilon}$ envoie $L^{1}$ dans $L_{\text {faible }}^{1}$.

Les preuves des résultats précédents sont, on l'a vu, fortement liées à la théorie des CZO. En fait, il y a presque toujours deux versants au moins d'un même résultat: un versant banachique et un versant théorie des opérateurs. Il est temps de passer de ce côte-ci.

\section{De l'Impossibilité d'un Calcul Symbolique Classique sur les CZO}

Nous allons reprendre, dans le cadre des opérateurs de Calderón-Zygmund, un programme introduit par R. Beals dans le cadre des opérateurs pseudodifférentiels.

Soit $Q \subset \mathscr{L}(H, H)$ une algèbre d'opérateurs continus sur un espace de Hilbert $H$. Dans les exemples qui nous intéressent, cette algèbre n'est pas fermée (pour la norme d'opérateur). La question du calcul symbolique consiste à savoir si tout opérateur $T \in \mathscr{Q}$ inversible dans $\mathcal{L}(H, H)$ est inversible dans $Q$. $R$. Beals a montré que c'est effectivement le cas pour les algèbres d'opérateurs pseudo-différentiels.

Nous allons voir que la réponse est non pour l'algèbre $A$ de Lemarié où $A$ est l'algèbre des $T \in \mathrm{CZO}$ tels que $T(1)=T^{*}(1)=0$.

Théorème 7. Pour tout $\epsilon \in] 0,1 / 2\left[\right.$, il existe $T \in A$ inversible sur $L^{2}$ et non inversible sur $L^{p}$ pour $|1 / p-1 / 2| \geqslant \epsilon$. En particulier, $T^{-1} \notin A$. 
Il existe également un opérateur $T \in A$ inversible sur tous les $L^{p}, 1<p<+\infty$, et tel que $T^{-1}$ ne soit pas un $\mathrm{CZO}$.

Le théorème résulte immédiatement des résultats du paragraphe précédent. En gardant les mêmes notations, et si $\epsilon \in] 0,1 / 2\left[\right.$ est fixé, soit $T_{\epsilon}$ l'opérateur défini par

$$
T_{\epsilon} f=\sum_{I}\left\langle f,\left(D^{-\alpha} \tau\right)_{I}\right\rangle\left(D^{-\alpha} \tau\right)_{I},
$$

où $\alpha=q-1 / 2-\epsilon$.

Grâce au lemme 5, on voit que $T_{\epsilon} \in A ; T_{\epsilon}$ est inversible sur $L^{2}$, et

$$
T_{\epsilon}^{-1}\left(\left(D^{-\alpha} \tau\right)_{I}\right)=\left(D^{\alpha} \sigma\right)_{I} .
$$

D'après le théorème $5, T_{\epsilon}$ est inversible sur $L^{p}$ exactement quand $|1 / p-1 / 2|<\epsilon$. Le choix $\alpha=q-1$ donne de la même manière la deuxième partie de l'énoncé.

Remarques. 1) L'inversibilité d'un opérateur quelconque $T$ sur $L^{2}$, supposé continu sur les $L^{p}$, entraine automatiquement l'existence d'un $\epsilon>0$ tel que $T$ soit inversible sur $L^{p}$ pour $|1 / p-1 / 2|<\epsilon$ (communication personnelle de G. David et S. Semmes).

2) Nos contre-exemples ont des noyaux assez peu réguliers, qui vérifient (28) et (29) avec $\delta=1 / 2$. P. G. Lemarié a construit des contre-exemples $T_{\epsilon}$ dont les noyaux vérifient, pour tous $p, q,\left|\partial_{x}^{p} \partial_{y}^{q} K(x-y)\right| \leqslant C_{p, q}|x-y|^{-p-q-1}$, et tels que $T_{\epsilon}\left(x^{n}\right)=T_{\epsilon}^{*}\left(x^{n}\right)=0$ pour tout $n$.

\section{Conclusion}

Bien que la théorie des CZO joue un rôle central dans l'étude des bases hilbertiennes ou inconditionnelles de $L^{2}$ du type de celles d'Y. Meyer, de Battle et Lemarié, ou des théorèmes 1 et 2 , la classe des opérateurs naturellement liés à la transformation en ondelettes, telle qu'elle est décrite dans le lemme 1, est strictement plus large que la classe des CZO de l'algèbre de Lemarié.

Du point de vue banachique, cela exprime que les bases de $L^{2}$ qu'on peut construire en discrétisant la transformation en ondelettes ne sont pas nécessairement des bases de $L^{p}, H^{1}$ ou BMO. La raison essentielle en est la différence de nature pouvant exister entre deux bases biorthogonales de $L^{2}$.

On aimerait comprendre d'où provient cette différence. Plus précisément, étant données des $\sigma_{I}$ formant une base inconditionnelle de $L^{2}$, localisées autour de l'intervalle $I$ en variable d'espace et autour des fréquences de module $1 /|I|$, que peut-on dire du système biorthogonal associé? 
En théorie des opérateurs, cette question peut par exemple devenir si $T$ est un CZO de l'algèbre de Lemarié inversible sur $H^{1}$ et BMO, est-ce que $T^{-1}$ est lui aussi un $\mathrm{CZO}$ ?

Je tiens à remercier très vivement Yves Meyer.

Le théorème 5 , en particulier, existe grâce aux discussions que nous avons eues. Et c'est l'ensemble de cet article qui doit beaucoup à sa chaleureuse attention.

\section{Bibliographie}

[1] Calderón, A. P. Commutators, singular integrals on Lipschitz curves and applications, Actes du Congrès d'Helsinki, 1978.

[2] Coifman, R. R. et Meyer, Y. Au-delà des opérateurs pseudo-différentiels. Astérisque, N. ${ }^{\circ} 57$.

[3] Coifman, R. R. et Meyer, Y. The discrete wavelet transform. Séminaire de Mathématique du Courant Institute.

[4] Daubechies, I. (Communication orale et texte en préparation).

[5] David, G., Journé, J. L., Semmes, S. Opérateur de Calderón-Zygmund et fonctions para-accrétives. Revista Matemática Iberoamericana, 1(4), (1985), 1-56.

[6] Grossman, A., Morlet, J. et Paul, T. Transforms associated to square integrable group representations, I. General results, J. Math. Phys., 26(10), October 1985, 2473-2479.

[7] Journé, J. L. Calderón-Zygmund operators, pseudo-differential equations and the Cauchy integral of Calderón, L.N. 994, Springer Verlag.

[8] Lemarié, P. G. Algèbres d'opérateurs et semi-groupes de Poisson sur un espace de nature homogène, Thèse de 3e cycle, 1984, Univ. Paris XI, Centre d'Orsay.

[9] Lemarié, P. G. et Meyer, Y. Ondelettes et bases hilbertiennes.

[10] McIntoch, A. et Meyer, Y. Algèbres d'opérateurs définis par des intégrales singulières, C.R. Acad. Sc., Paris.

[11] Meyer, Y. Remarques sur un théorème de J. M. Bony, Suppl. Rend. Circ. Mat. Palermo, 1(1981), 1-20.

[12] Meyer, Y. Minimalité de certains espaces fonctionnels et applications à la théorie des opérateurs. Séminaire Bony-Sjöstrand-Meyer, 1984-1985, Centre de Mathématiques, Ecole Polytechnique.

[13] Meyer, Y. La transformation en ondelettes et les nouveaux paraproduits, A paraître aux Actes du Colloque d'Analyse non linéaire du CEREMADE, Univ. Paris-Dauphine.

[14] Meyer, Y. Principe d'incertitude, bases hilbertiennes et algèbrés d'opérateurs, Séminaire Bourbaki, 38ème année, 1985-86, N. ${ }^{\circ} 662$.

[15] Tchamitchian, P. Calcul symbolique sur les opérateurs de Calderón-Zygmund et bases inconditionnelles de $L^{2}(\mathbb{R}), C$. R. Acad. Sci., Paris, t. 303, Série 1, N. ${ }^{\circ} 6,1986$.

\section{Philippe Tchamitchian}

Centre de Physique Théorique, et Service de Mathématiques

C.N.R.S., case 907 , 13288 Marseille Cedex 9

Faculté S. -Jérôme

13397 Marseille Cedex 13 